\title{
The possible role of SRMS in colorectal cancer by bioinformatics analysis
}

\author{
Jie Zhang ${ }^{1}$, Weidong Liu', Sisi Feng ${ }^{2}$ and Baiyun Zhong ${ }^{\text {** }}$
}

\begin{abstract}
Background: Src-related kinase lacking C-terminal regulatory tyrosine and N-terminal myristoylation sites (SRMS) is a non-receptor tyrosine kinase that has been found to be overexpressed in various tumors. However, the role of SRMS in colorectal cancer (CRC) has not been well established.

Methods: We evaluated the expression levels of SRMS in CRC using GEPIA, Oncomine, and HPA datasets. Survival information and gene expression data of CRC were obtained from The Cancer Genome Atlas (TCGA). Then, the association between SRMS and clinicopathological features was analyzed using UALCAN dataset. LinkedOmics was used to determine co-expression and functional networks associated with SRMS. Besides, we used TISIDB to assess the correlation between SRMS and immune signatures, including tumor-infiltrating immune cells and immunomodulators. Lastly, protein-protein interaction network (PPI) was established and the function enrichment analysis of the SRMSassociated immunomodulators and immune cell marker genes were performed using the STRING portal.
\end{abstract}

Results: Compared to normal colorectal tissues, SRMS was found to be overexpressed in CRC tissues, which was correlated with a poor prognosis. In colon adenocarcinoma (COAD), the expression levels of SRMS are significantly correlated with pathological stages and nodal metastasis status. Functional network analysis suggested that SRMS regulates intermediate filament-based processes, protein autophosphorylation, translational initiation, and elongation signaling through pathways involving ribosomes, proteasomes, oxidative phosphorylation, and DNA replication. In addition, SRMS expression was correlated with infiltrating levels of CD4+ T cells, CD56dim, MEM B, Neutrophils, Th2, Th17, and Act DC. The gene ontology (GO) analysis of SRMS-associated immunomodulators and immune cell marker genes showed that they were mainly enriched in the immune microenvironment molecule-related signals. Kyoto Encyclopedia of Genes and Genomes (KEGG) enrichment analysis of these genes indicated that they are involved in multiple cancer-related pathways.

Conclusions: SRMS is a promising prognostic biomarker and potential therapeutic target for CRC patients. In particular, SRMS regulates CRC progression by modulating cytokine-cytokine receptor interaction, chemokines, IL-17, and intestinal immune networks for IgA production signaling pathways among others. However, more studies are needed to validate these findings.

Keywords: Srms, Colorectal cancer, Immune signatures, Bioinformatics analysis

*Correspondence: xycsuhn@163.com

${ }^{1}$ Department of Clinical Laboratory, Xiangya Hospital, Central South University, 87 Xiangya Road, Kaifu District, Changsha 410008, China

Full list of author information is available at the end of the article

\section{Introduction}

Globally, CRC is one of the most prevalent malignancies and the fourth leading cause of cancer-related mortality, resulting in almost 900,000 annual mortality [1]. The high incidence of colorectal cancer is correlated with age, diet, race, lifestyle, genetic alteration, and other factors. Since the early symptoms of colorectal cancer are not typical, original author(s) and the source, provide a link to the Creative Commons licence, and indicate if changes were made. The images or other third party material in this article are included in the article's Creative Commons licence, unless indicated otherwise in a credit line to the material. If material is not included in the article's Creative Commons licence and your intended use is not permitted by statutory regulation or exceeds the permitted use, you will need to obtain permission directly from the copyright holder. To view a copy of this licence, visit http://creativecommons.org/licenses/by/4.0/. The Creative Commons Public Domain Dedication waiver (http://creativeco mmons.org/publicdomain/zero/1.0/) applies to the data made available in this article, unless otherwise stated in a credit line to the data. 
about $25 \%$ of colorectal cancers present distant metastasis at the time of initial diagnosis [2]. Currently, the major therapeutic options for CRC include surgery, chemotherapy, radiotherapy, biotherapy, and immunotherapy. However, the 5-year survival outcomes for advanced CRC patients are approximately $10 \%[3,4]$. Identification of genes associated with tumor formation and metastasis will provide new ideas and targets for anti-CRC therapy.

Src-related kinase lacking C-terminal regulatory tyrosine and $\mathrm{N}$-terminal myristoylation sites (SRMS) encode a 53-kDa non-receptor tyrosine kinase protein that was first cloned in mouse neural precursor cells [5]. SRMS is composed of 488 amino acids, which has a similar structure to Src family kinases. Its functional regions are composed of a Src homology 3 (SH3) domain, a Src homology 2 (SH2) domain, and a protein kinase domain [6]. These domains are important in mediating a series of intra-molecular or inter-molecular interactions as well as downstream signal transductions. The non-receptor tyrosine kinase plays an important role in the regulation of cell growth, proliferation, and invasion by activating the downstream substrate to initiate tyrosine phosphorylation [7]. Compared with other members of the nonreceptor tyrosine kinase family, the roles and functions of SRMS are still in the early stages. A recent review article noted that SRMS was overexpressed in six breast cancer cell lines and its levels were elevated in breast tumors compared to adjacent normal tissues [8]. In addition, a proteomic study of gastric cancer patients showed that SRMS was the only differentially expressed kinase [9]. However, the expression levels and biological functions of SRMS in CRC have not been clearly elucidated.

In this study, we evaluated and validated the expression levels and prognosis value of SRMS in CRC through multiple independent cohorts. Then, the relationship between SRMS expression levels and clinic-pathological features such as histological grade and metastasis were systemically determined. In addition, we performed coexpression analysis and assessed the gene sets associated with SRMS in CRC through gene set enrichment analysis (GSEA). Lastly, we assessed the roles of SRMS in tumor immunity.

\section{Materials and methods}

\section{SRMS differential expression and proggesnosis analysis}

GEPIA (http://gepia.cancer-pku.cn/) is a database used to perform comprehensive and customizable functions using TCGA and GTEx data [10]. It includes 9736 tumor and 8587 normal samples. We used GEPIA to analyze the mRNA expression levels of SRMS in tumor and normal CRC tissue samples. The $Y$-axis represents the average $\log 2$ abundance in transcripts-per-million (TPM). DNA copy number variations $(\mathrm{CNV})$ of SRMS in CRC and normal tissues were examined using the Oncomine 4.5 database. Oncomine (https://www.oncomine.org/) is the largest cancer microarray database and data-mining platform [11]. It contains 715 datasets and 86,733 samples. The screening conditions were set as (1) gene: "SRMS";(2) analysis type: "cancer VS normal analysis"; (3) cancer type: "Colorectal cancer"; (4) data type: "mRNA"; and (5) $P$ value $<0.05$, fold change $>2$, gene rank $=$ top $10 \%$.

Subsequently, we compared SRMS protein expression levels between CRC tissues and normal colorectal tissues in the Human Protein Atlas (HPA) database. The HPA database (https://www.proteinatlas.org/) is aimed at mapping $\mathrm{IHC}$-based protein expression profiles in cancerous and normal tissues as well as in cell lines [12]. Protein expression score is based on immunohistochemical data manually scored with regard to staining intensity (negative, weak, moderate, or strong) and fraction of stained cells $(<25 \%, 25-75 \%$, or $>75 \%)$. For prognostic analysis, the gene expression profiles and clinical information for CRC patients were obtained from the TCGA database. Kaplan-Meier survival curves were plotted with R 3.6.3.

\section{UALCAN analysis}

UALCAN (http://ualcan.path.uab.edu/) is a comprehensive and interactive web-portal that provides an easy access to publicly available cancer OMICS data [13]. Moreover, it has gene expression profiles and patient survival information. In this study, SRMS expression levels in various sub-groups of clinical characteristics (age, gender, race, stages, histological types, and nodal metastasis status) were examined in UALCAN. $p \leq 0.05$ was considered statistically significant.

\section{LinkedOmics analysis}

LinkedOmics (http://www.linkedomics.org/login.php) is a web-based platform that includes multiple omics data and proteomics data based on mass spectrometry (MS) generated by the CPTAC [14]. The "LinkFinder" module of LinkedOmics was used to evaluate gene co-expressions that are related to SRMS in the TCGA CRC cohort $(n=379)$. Statistical analyses were conducted using Pearson's correlation coefficients and results were presented in form of heatmaps, scatter plots, and volcano plots. The expression of co-expressed genes was normalized using $Z$-score approach, $Z=(X$-mean $(X)) /$ sd $(X)$. Based on the median SRMS gene expression, the data were divided into two groups, high expression group and low expression group. The color of the bar indicates the redder the color, the higher the gene expression; the bluer the color, the lower the gene expression.

In the "LinkInterpreter" module of LinkedOmics, we also performed functional enrichment analysis of 

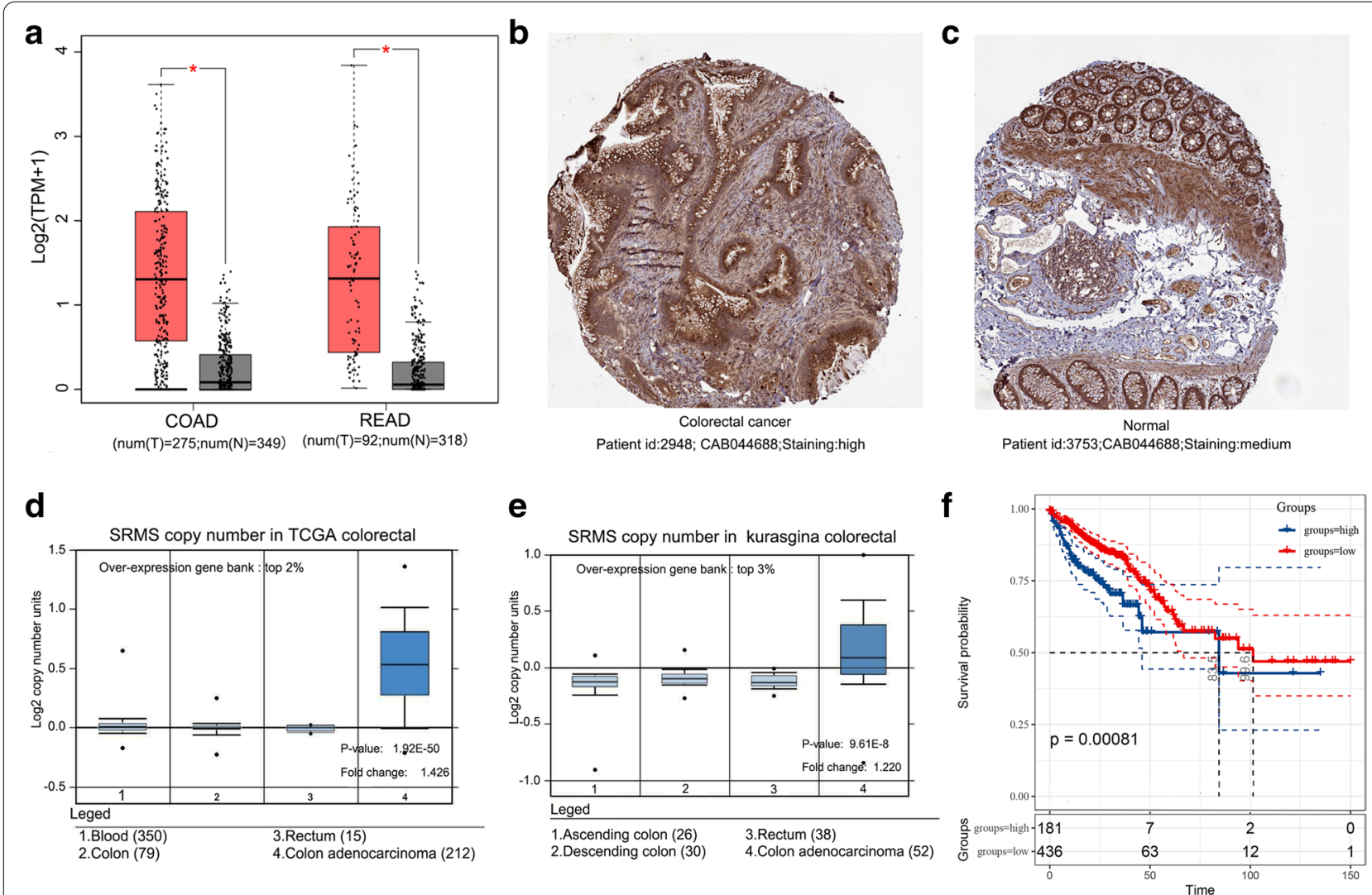

Fig. 1 Expression and prognostic value of SRMS in CRC. A Comparison of SRMS mRNA expression levels between normal and tumor tissues in the TCGA and GTEx CRC cohorts, $p$ value cutoff $<0.01$, |Log2FC| Cutoff $<1$. B Protein expression levels of SRMS in CRC tissues from the HPA database. C Protein expression levels of SRMS in non-cancerous colorectal tissues. D SRMS copy numbers in the Cancer Genome Atlas (TCGA) Colorectal datasets. ${ }^{*}, p<0.05$. (E) SRMS copy numbers in Kurashina colorectal datasets. ${ }^{*}, p<0.05$. F Kaplan-Meier survival curves reveal the prognostic value of SRMS in CRC

co-expressed genes. Data from LinkFinder were used to perform GO and KEGG pathway enrichment analysis through GSEA. Rank criterion was an FDR $<0.05$ and 500 simulations.

\section{TISIDB analysis}

TISIDB (http://cis.hku.hk/TISIDB/) is a web portal for tumor and immune system interactions, including genomics and transcriptomics of 30 cancer types from TCGA, RNA sequencing data set of patient cohorts treated with immunotherapy [15]. The TCGA database provides a large amount of tumor public data, providing useful information for studying the complex interaction of the tumor microenvironment [16]. TISIDB has "Function," "Literature," "Screening," "Immunotherapy," "Lymphocyte," "Immunomodulator," "Chemokine," "Subtype," "Clinical", and "Drug" modules. We used the TISIDB database to determine the correlations between SRMS expression levels and tumor-infiltrating lymphocytes (TILs) and immunomodulators.

\section{STRING analysis}

STRING (https://string-db.org/) is a database of known and predicted protein-protein interactions [17]. Currently, it has $24,584,628$ proteins from 5090 organisms. In this study, STRING was used to visualize protein-protein interaction networks and to predict the functions of the SRMS-associated immunomodulators and immune cell marker genes. GO and KEGG enrichment analysis was performed using Cluster-Profiler package 3.14.0. Proteins with a minimum required interaction score greater than or equal to 0.400 .

\section{Result}

SRMS expression and prognosis in colorectal cancer Initially, we compared mRNA expression levels of SRMS in GEPIA, which matched TCGA and GTEx data. mRNA 

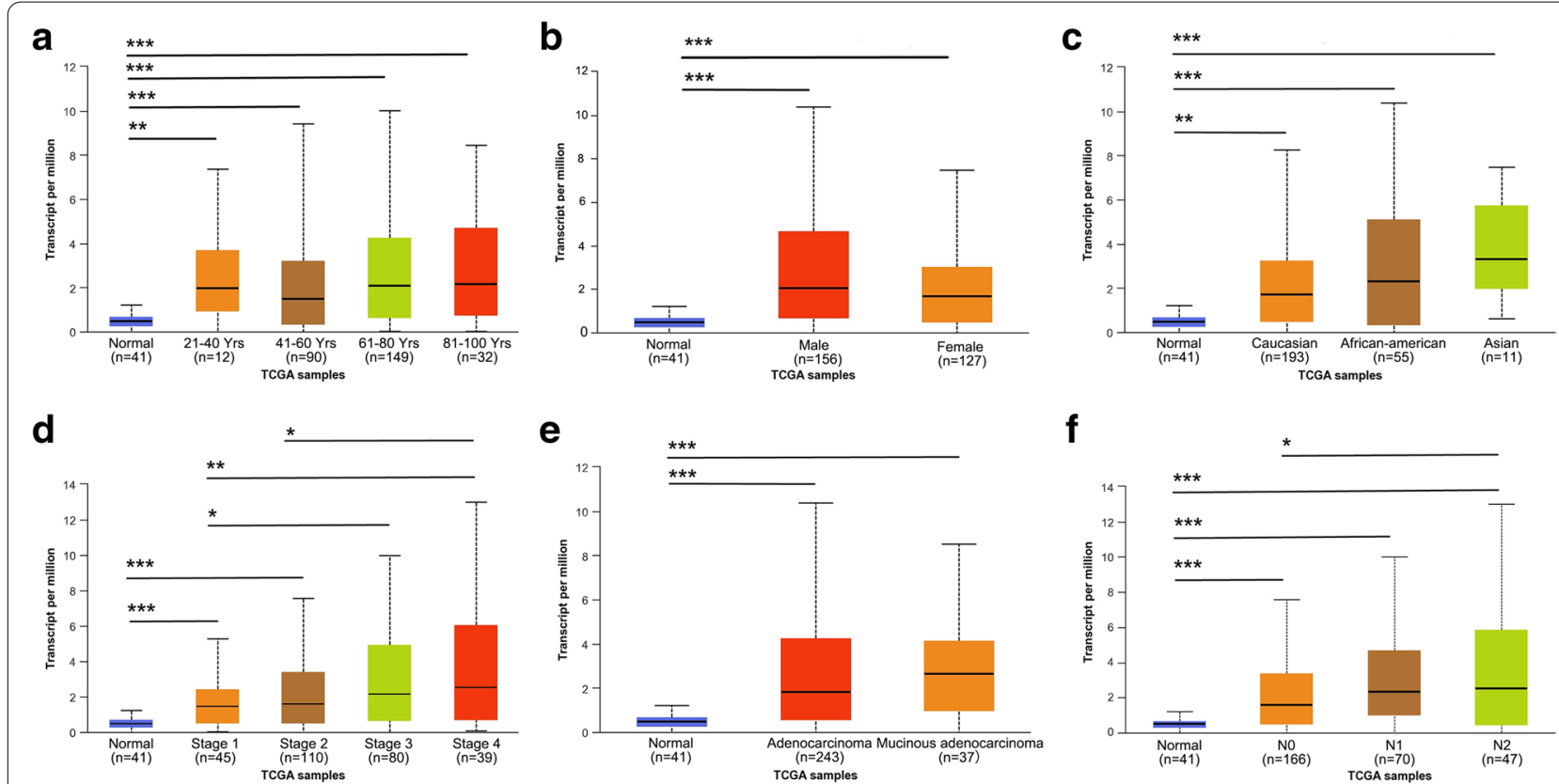

Fig. 2 SRMS transcription levels in subgroups of patients with colon adenocarcinoma. A age, B gender, $\mathbf{C}$ race, D clinical stages, E histological types, F nodal metastasis status. Data are mean \pm SE. ${ }^{*}, p<0.05 ; *^{* *}, p<0.01 ;{ }^{* * *}, p<0.001$

expression levels of SRMS were significantly elevated in CRC tissues than in the paired adjacent normal tissues $(p \leq 0.05$, Fig. 1A). Moreover, from the HPA database, protein expression levels of SRMS were high elevation in tumor tissues and moderate in normal tissues (Fig. 1B, C). Data in the Oncomine database, including TCGA colorectal and Kurasgina colorectal, showed that CNVs of SRMS were significantly elevated in colon adenocarcinoma (COAD) tissues than in paired normal tissues ( $p$ $<0.01$, Fig. 1D, E). KM-Plotter shows that SRMS overexpressed is associated with a poor prognosis (Fig. 1F). It is worth noting that a more clear difference can be found between the overall survivals rates within 2000 days among the two groups. Therefore, SRMS expression is a potential diagnostic and prognostic indicator for CRC.

\section{SRMS expression in clinical characteristic sub-groups}

The association between SRMS and clinicopathological features was further evaluated in the online cancer OMICS database of UALCAN. In subgroup analyses based on age, gender, race, clinical stage, and histological and nodal metastasis status, the transcription level of SRMS was significantly elevated in CRC patients than in healthy individuals ( $p<0.05$, Figs. 2 and 3). In COAD patients, SRMS expression levels were positively correlated with clinical stages and nodal metastasis. The expression of SRMS in stage 4 was higher than in stage 1 and $2(p=0.0028,0.015$, respectively). The expression of SRMS in N2 was high than in N0 $(p<0.05)$. In rectum adenocarcinoma (READ) patients, SRMS expression was negatively correlated with nodal metastasis. The expression of SRMS in N2 was low than in N0 $(p<0.05)$.

\section{SRMS co-expression networks in colorectal cancer}

To clarify on the functional properties of SRMS in CRC, the "LinkFinder" module in LinkedOmics was used to analyze the co-expression networks of SRMS. As shown in the volcano plot (Fig. 4A), a total of 2176 genes (red dots) were significantly positively correlated with SRMS while 1368 genes (green dots) were significantly negatively correlated $(p<0.05)$. The left and right heatmaps show the top 50 genes that were positively and negatively correlated with SRMS, respectively (Fig. 4B, C). SRMS expression was positively correlated with the expression of C20orf195 (positive rank \#1, $r=0.389, p=3.90 \mathrm{E}-15$ ), HES2 $(r=0.301, p=2.20 \mathrm{E}-9)$, PTK6 $(r=0.300, p=3.04 \mathrm{E}-$ $9)$, and SYNGR3 $(r=0.300, p=2.43 \mathrm{E}-9)$, and negatively correlated with the expression of PNRC2 (negative rank \#1, $r=-0.247, p=1.16 \mathrm{E}-6)$, STMN1 $(r=-0.246$, $p=1.20 \mathrm{E}-6)$, and EPHX1 $(r=-0.234, p=4.04 \mathrm{E}-6)$.

GO biological process analysis by GSEA showed that co-expressed genes were mainly involved in the intermediate filament-based processes, epidermis development, and protein autophosphorylation (Fig. 4D). In contrast, translational initiation and elongation, mitochondrial gene expression, and cytoplasmic translation were inhibited. KEGG pathway analysis by GSEA showed enrichment in inositol phosphate metabolism, ribosomes, 

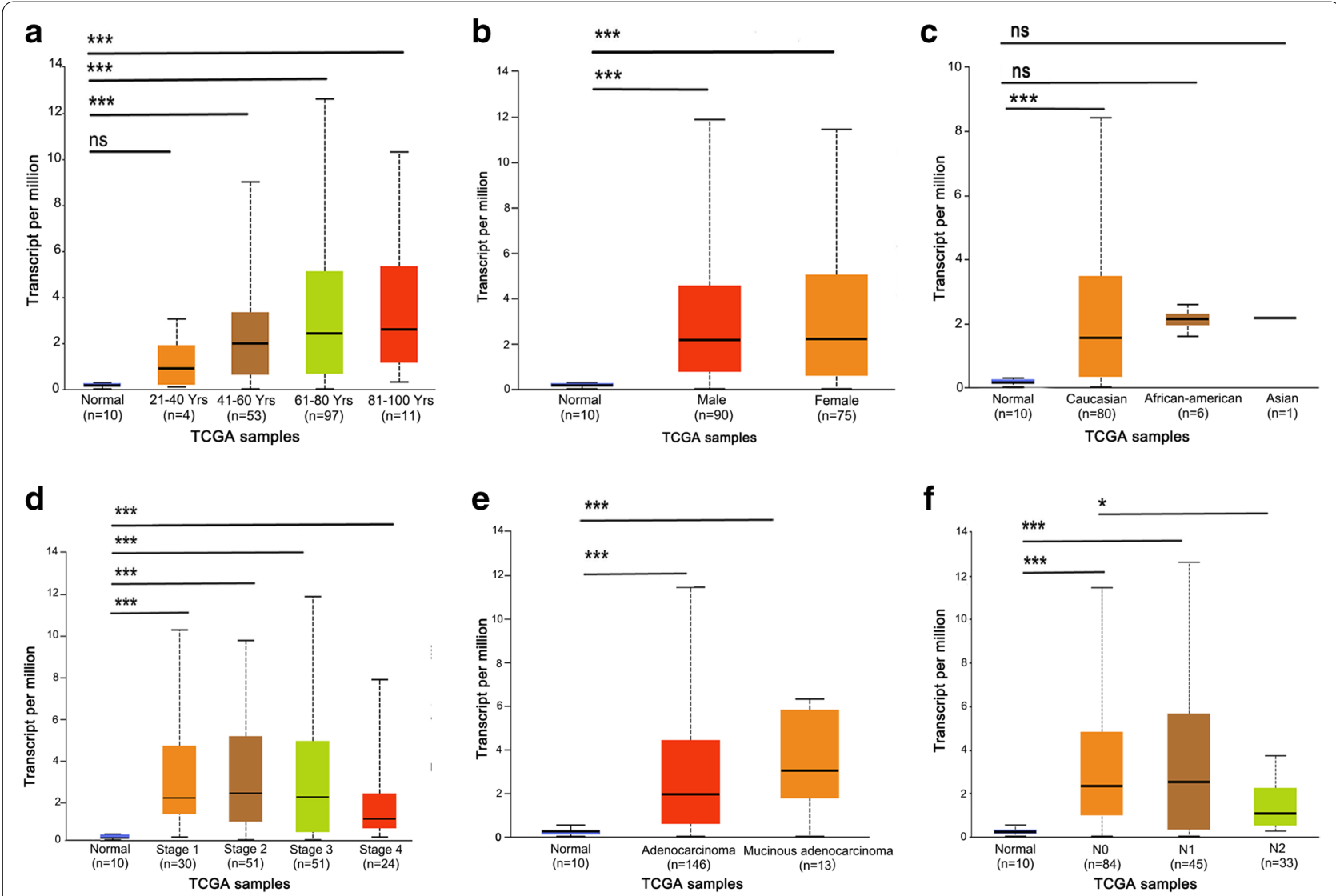

Fig. 3 SRMS transcription levels in subgroups of patients with rectum adenocarcinoma. A age, B gender, C race, D clinical stages, E histological types, $\mathbf{F}$ nodal metastasis status. Data are mean \pm SE. ${ }^{*}, p<0.05 ;{ }^{* *}, p<0.01 ;{ }^{* * *}, p<0.001 ; n$ (non-significant), $p>0.05$

proteasomes, oxidative phosphorylation, and DNA replication among others (Fig. 4E).

\section{Association between SRMS and immune signatures}

Figure 5 shows that some immune subsets were associated with SRMS mRNA expression levels in COAD and READ. The 7 types of tumor-infiltrating lymphocytes that exhibited important correlations with SRMS in COAD included activated CD4 $+\mathrm{T}$ cells (Act CD4; Spearman: $r=-0.196, p=2.37 \mathrm{e}-05$ ), CD56 dim natural killer cells (CD56 dim, Spearman: $r=0.283, p=7.68 \mathrm{e}-10$ ), memory B cells (MEM B, Spearman: $r=-0.115, p=0.0137$ ), neutrophils (neutrophil, Spearman: $r=0.102, p=0.0293$ ), effector memory CD4+ T cells (Tem CD4, Spearman: $r=-0.296, p=1.17 \mathrm{e}-10)$, type $2 \mathrm{~T}$ helper cells (Th2, Spearman: $r=-0.187, p=5.98 \mathrm{e}-05)$, and type $17 \mathrm{~T}$ helper cell (Th17, Spearman: $r=0.23, p=6.6 \mathrm{e}-07$ ).

The 5 types of tumor-infiltrating lymphocytes that exhibited important correlations with SRMS in READ included activated dendritic cells (Act DC, Spearman: $r=0.23, p=6.6 \mathrm{e}-07), \mathrm{CD} 56 \mathrm{dim}$ natural killer cells
(CD56 dim, Spearman: $r=0.214, p=0.006$ ), effector memory CD4 T cells (Tem CD4, Spearman: $r=-0.188$, $p=0.011$ ), type $2 \mathrm{~T}$ helper cells (Th2, Spearman: $r=-0.187, p=0.0213$ ), and type $17 \mathrm{~T}$ helper cells (Th17, Spearman: $r=0.178, p=0.021$ ).

Moreover, we identified 19 immunostimulators (C10orf54, CD276, CD28, CD70, CXCL12, ENTPD1, IL6R, KLRK1, RAET1E, TMRM173, TMIGD2, ULBP1, TNFRSF13B, TNFRSF14, TNFRSF18, TNFRSF25, TNFRSF4, TNFSF4, and TNFSF9) and 8 immuno-inhibitors (ADORA2A, BTLA, CD160, KDR, LGALS9, PVRL2, TGFB1, and TGFBR1) that were significantly associated with SRMS in CRC (Fig. 6A, B).

\section{Immune signatures associated with the SRMS gene}

The SRMS-associated 27 immunomodulators and 191 immune cell marker genes were analyzed in the STRING database to validate functional connectivity. From the STRING database, we obtained the SRMSassociated immune gene PPI network (enrichment $p$ value $<1.0 \mathrm{e}-16$ ) of a total of 214 nodes and 829 edges, which represented proteins and functional interactions 
(Fig. 6C). Next, we performed GO and KEGG enrichment analysis for these genes. The GO terms within the biological processes (BP), cell components (CC), and molecular function (MF) categories are shown in Fig. 6E. GO analysis showed that these genes were mainly enriched in the immune microenvironment moleculerelated signals. Consequently, we probed the signaling pathways through which SRMS regulates immune responses in CRC. Figure 6D shows the top 20 enriched KEGG pathways, including cytokine-cytokine receptor interaction, chemokine, Th17 cell differentiation, IL-17, and intestinal immune network for IgA production. These signaling pathways may be related to SRMS-mediated immune events.

\section{Discussion}

It is important to understand the basic mechanism of cancer occurrence at the gene level for increasing the efficiency of cancer therapy. In the present study, SRMS was highly expressed in CRC tissues compared to paired normal tissues.

A higher SRMS expression level was significantly associated with late TNM stages, more lymph node metastasis in COAD, but not with age, gender, race, histological. In READ, there was no significant association between the SRMS expression and patients' age, gender, race, clinical stage, and histological. Moreover, SRMS expression was correlated with many immune cells and immunostimulators, and these immune signatures were focused on inflammation and cancer signaling pathways.

Tumor occurrence is closely associated with cell proliferation, differentiation, and apoptosis. Non-receptor tyrosine kinases, including PTK6 (protein tyrosine kinase 6), FAK (focal adhesion kinase), and Jak (janus kinase) are involved in cell differentiation, apoptosis, and proliferation through their interactions with transmembrane receptors [18]. PTK6 is the most studied member of the non-receptor tyrosine kinase family. Important roles of PTK6 in various cancers, including breast, prostate, and colon cancers have been reviewed [19]. SRMS and PTK6 genes are closely linked on the chromosome. Additionally, SRMS has been shown to biochemically interact with PTK6, which phosphorylates the C-terminus of PTK6 [20]. It has been reported that PTK6 expression is highest in normal colon epithelial tissues and decreases during colon tumor progression [21].

In the present study, we found that proteins, CNVs, and mRNA expression levels of SRMS were significantly elevated in CRC than in normal colorectal tissues. Copy number variants play a significant role in genetic variations and evolution, and can also cause genetic diseases and cancer [22]. We also found that elevated SRMS expression levels were correlated with poor prognostic outcomes, which may be involved in CRC progression. In addition, high expression levels of SRMS have been associated with advanced clinical stage and lymph node metastasis in COAD. However, SRMS expression was negatively correlated with nodal metastasis in READ. There are probably several reasons for this pattern: (1) The differences in anatomical location and biological function between COAD and READ. Studies have shown that right colon cancer patients had significantly higher gene mutation than left colon and rectum cancer patients [23]; (2) With lymph node metastasis, the exertion effects of some tumor suppressor factors reduce the expression of SRMS; (3) The number of cases with READ in UALCAN is relatively small. A larger sample size would obtain more robust and reliable statistic. Therefore, more clinical cohorts are needed to validate SRMS as a diagnostic or prognostic marker in CRC.

Within the gene co-expression network of SRMS, we identified genes that were significantly positively correlated, such as C20orf195, HES2, PTK6, and SYNGR3. C20orf195, also known as fibronectin type III domaincontaining 11, is mainly expressed in the testis. Regrettably, its role in human disease is still unknown. HES genes are Notch downstream target genes, which could reflect expression levels of Notch signals [24]. Aberrant activation of Notch signaling has been associated with various tumors, such as breast and colorectal cancers $[25,26]$. As mentioned earlier, PTK6 and SRMS have a close relationship. PTK6 is mainly expressed in epithelial tissues, with the highest levels in gastrointestinal linings [27]. In the azoxymethane model, PTK6 promoted STAT3 activation to promote survival and proliferation of damaged cells and colon tumorigenesis [28]. SYNGR3 is a synaptic vesicle-associated protein that interacts with the dopamine transporter. There are studies indicating that SYNGR3 may serve as a potential biomarker for the diagnosis and treatment of malignant tumors $[29,30]$.

We also found that SRMS expression was negatively associated with PNRC2, STMN1, and EPHX1 levels in the co-expression network. PNRC2, belonging to the PNRC family, was first found in breast cancer. In colorectal cancer, PNRC2 is expressed at low levels and PNRC2 upregulated can inhibit cell proliferation, migration, invasion, and EMT [31]. STMN1 is a major cytosolic phosphoprotein that regulates cell spindle formation and microtubule dynamics. Overexpression of STMN1 blocks the invasion of cancer cells and induces cancer cells growth arrest at the G2/M phase checkpoint [32, 33]. EPHX1, which mainly localizes in the endoplasmic reticulum, is a biotransformation enzyme. However, EPHX1 has been less studied in tumors. It is considered to be well associated with the anti-epileptic drug resistance [34]. 
(See figure on next page.)

Fig. 4 SRMS co-expression genes in CRC. A SRMS correlated genes in CRC were analyzed by the Pearson test. Red dots represent positively correlated genes dots while green represent negatively correlated genes. B Heatmaps of the top 50 genes that were positively correlated with SRMS. C Heatmaps of the top 50 genes that were negatively correlated with SRMS. D GO annotations: biological processes of SRMS co-expression genes. E KEGG pathways analysis of SRMS co-expression genes

In addition, co-expression functional networks were found to be mainly involved in protein autophosphorylation, translational initiation and elongation, mitochondrial gene expression, and cytoplasmic translation. Thus, the SRMS co-expression network is involved in posttranscriptional regulation, which is closely associated with protein translation and phosphorylation.

KEGG analysis showed the SRMS co-expression genes mainly enrichment in the ribosomal, proteasomal, oxidative phosphorylation, and DNA replication pathways. Ribosomes, comprised of ribonucleoprotein and noncoding ribosomal RNAs in eukaryotes, are conserved molecular structures required for protein synthesis [35]. Cancer development and progression are associated with ribosomal dysregulation, which affects the expression of key factors involved in tumorigenesis [36]. It has been reported that a single ribosomal assembly factor promotes lung adenocarcinoma progression through the Notch signaling pathway [37]. The proteasome pathway is one of the major mechanisms of protein degradation. Among these mechanisms, 26S proteasomes are the most active isoforms that are involved in cell cycle progression, apoptosis, and transcription [38]. Therefore, they are potential targets for anticancer therapy. Suppressed oxidative phosphorylation is a basic feature of tumor cells and tumors [39]. Huang et al. showed that LYRM2 directly interacts with complex I and enhances its activity, thereby promoting oxidative phosphorylation to induce colorectal cancer cell growth [40]. Since co-expressed genes share functions and affect each other, SRMS may play a role in CRC occurrence and progression through the above factors.

In the TISIDB database, we found that the Reactome pathway of SRMS was mainly involved in the immune system. Moreover, SRMS expression levels were positively correlated with infiltration levels of CD56dim, neutrophils, and Th17 and negatively correlated with Act CD4, MEM B, Tem CD4, and Th2 in CRC. There were studies that some TILs can promote lymph node invasion [41]. Moreover, tumor-infiltrating immune cell patterns are associated with cancer initiation and prognosis. For instance, neutrophils and Th17 have been shown to correlate with a poor prognosis in colorectal carcinoma [42, 43]. Given that TILs are critical for immunosurveillance and immunotherapy, SRMS potentially serves as a promising target to shape the immune microenvironment in colorectal cancer. In addition, we identified the immunomodulators that were significantly associated with SRMS in CRC. Tumor cells utilize various immune escape mechanisms, creating a microenvironment that is favorable for tumor growth and metastasis [44]. Immunomodulators have the potential for cancer treatment.

The biological functions of these SRMS-associated immune genes were explored using GSEA enrichment analyses. The enrichment of GO biological processes suggested that these genes mainly regulated immune microenvironment molecules by affecting their activation, migration, and adhesion, etc. Moreover, cell components showed that these genes were enriched in various membranes such as secretory granule membrane and anchored component of membrane. In addition, molecular function analysis showed that these genes chiefly regulate cytokines, receptor, and bind with various structures, such as carbohydrate, chemokine, and tumor necrosis factor receptor superfamily.

KEGG pathway analysis of SRMS-associated immunomodulators and immune cell marker genes revealed that cytokine-cytokine receptor interaction, chemokine, Th17 cell differentiation, IL-17, and intestinal immune network for IgA production may be involved in SRMSmediated immune responses. Cytokine-cytokine receptor interaction is related to the viability and apoptosis rate of colon cancer cell lines [45]. It has been reported that the CXCL5 chemokine enhances the migratory and invasive properties of colorectal cancer cells by inducing epithelial-mesenchymal transition [46]. Chemokine signaling systems play critical roles in either the promotion or inhibition of tumor growth, proliferation, angiogenesis, or metastasis [47]. Aberrated activation of intestinal immune networks for IgA production signaling pathway promotes tumorigenesis [48]. IL-17 promotes tumor development through chronic tissue inflammation signals. In ETBF-colonized Min-CD $4^{\text {Stat3-/- }}$ mice, IL17 blockade can significantly reduce colon cancer formation [49]. For many cancers, Th17-cell signatures (RORC, IL17, IL23, STAT3) are correlated with the worse clinical outcomes [50]. Therefore, it is biologically plausible that SRMS promotes tumor immunity by regulating multiple signaling pathways. This study provides the first evidence for the link between SRMS and tumor immunity, opening up more avenues for CRC research. 
a SRMS association result

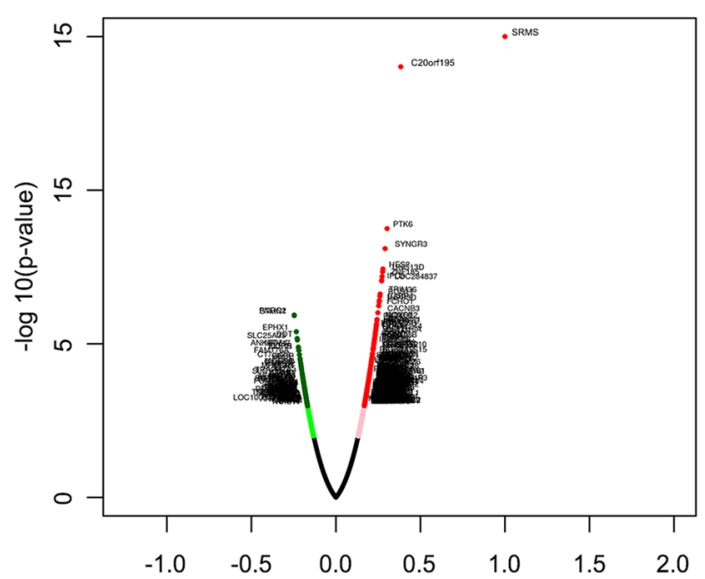

Pearson correlation coefficient ( pearson test)

b

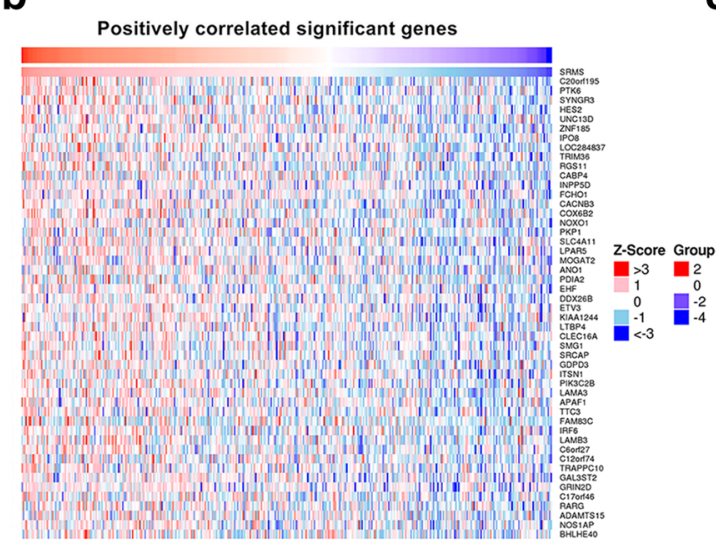

C

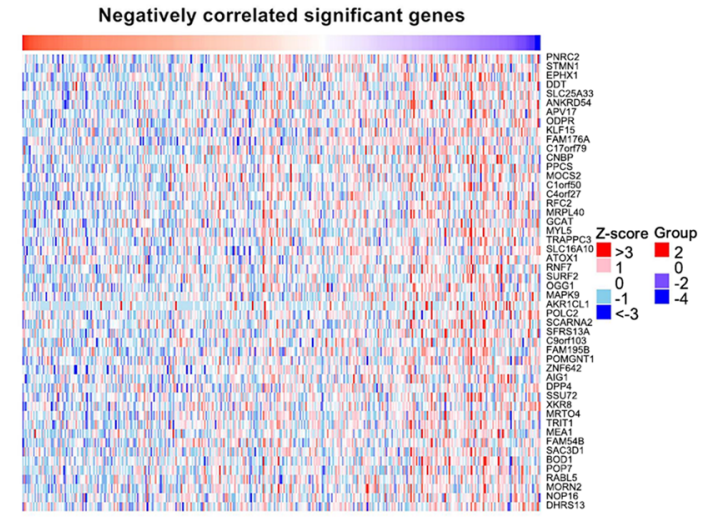

e

d

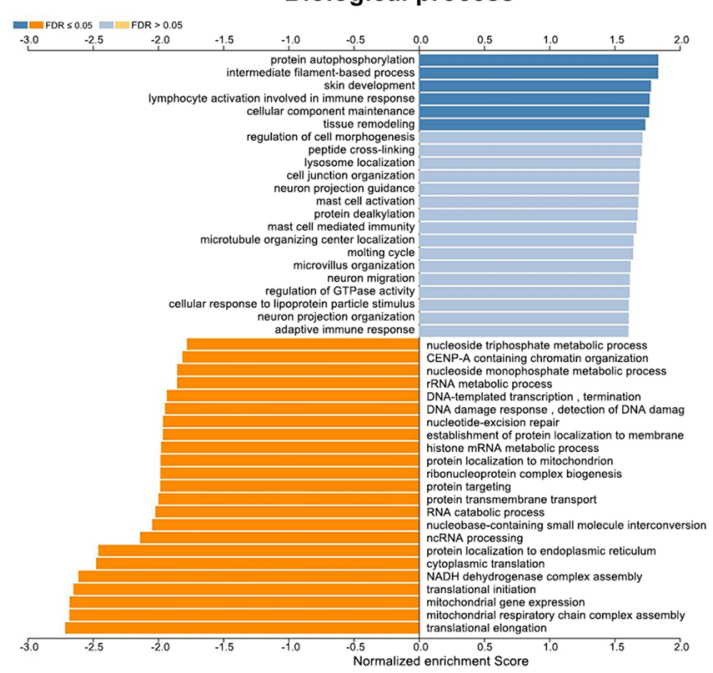

KEGG

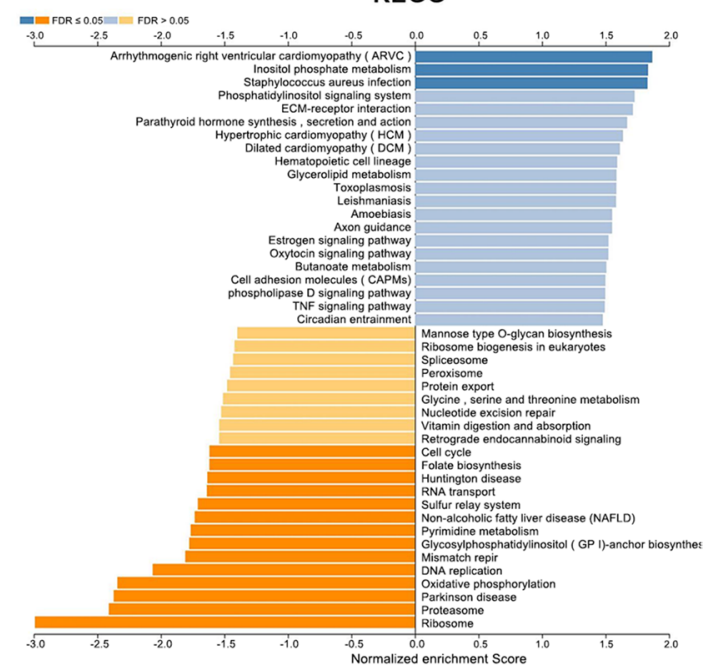

Fig. 4 (See legend on previous page.) 

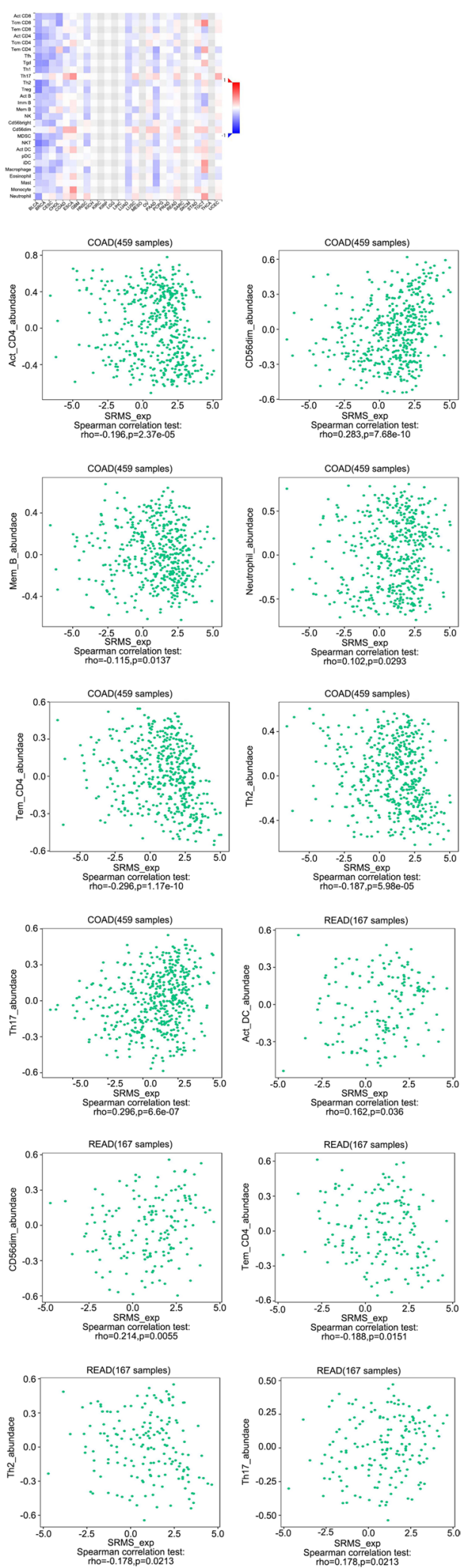

Fig. 5 Correlations between SRMS expression levels and tumor-infiltrating lymphocytes. Dot plots show the correlations between SRMS expression levels and tumor-infiltrating lymphocytes in COAD and READ cohorts

This study has several limitations. First, the data we analyzed were obtained from several public datasets, which is a lack of validation data. Second, the mechanisms of SRMS-mediates tumor immunity were not fully evaluated. More clarification and basic data are required to better assess the potential relationships between SRMS and CRC.

\section{Conclusion}

In summary, the present study demonstrated for the first time that SRMS is overexpressed and associated with an adverse clinical outcome in CRC. Moreover, SRMS expression levels were significantly correlated with various immune signatures. These findings indicate that SRMS might play a role in the control of tumor immune microenvironments.

\section{Abbreviations}

SRMS: Src-related kinase lacking C-terminal regulatory tyrosine and N-terminal myristoylation sites; CRC: Colorectal cancer; GO: Gene ontology; TILs: Tumorinfiltrating lymphocytes; COAD: Colon adenocarcinoma; READ: Rectum adenocarcinoma; GSEA: Gene set enrichment analysis; KEGG: Kyoto Encyclopedia of Genes and Genomes; Act CD4: Activated CD4+ T cells; CD56 dim: CD56 dim natural killer cells; MEM B: Memory B cells; Tem CD4: Effector memory CD4+ T cells; Th2: Type 2T helper cells; Th17: Type 17T helper cell; Act DC: Activated dendritic cells.

\section{Acknowledgements}

Not applicable.

\section{Authors' contributions}

$J Z$ wrote the manuscript and produced the Figs. SF contributed to the literature search for the manuscript. DL and $Y Z$ grammar edited the manuscript. All authors read and approved the manuscript for publication.

\section{Funding}

This work is supported by the Key Research and Development Program of Hunan Province (No.2015GK3002).

\section{Availability of data and materials}

All data supporting the conclusion of this article are included within the article.

\section{Declarations}

Ethics approval and consent to participate

Not applicable.

\section{Consent for publication}

Not applicable.

\section{Competing interests}

The authors declare that they have no competing interests. 

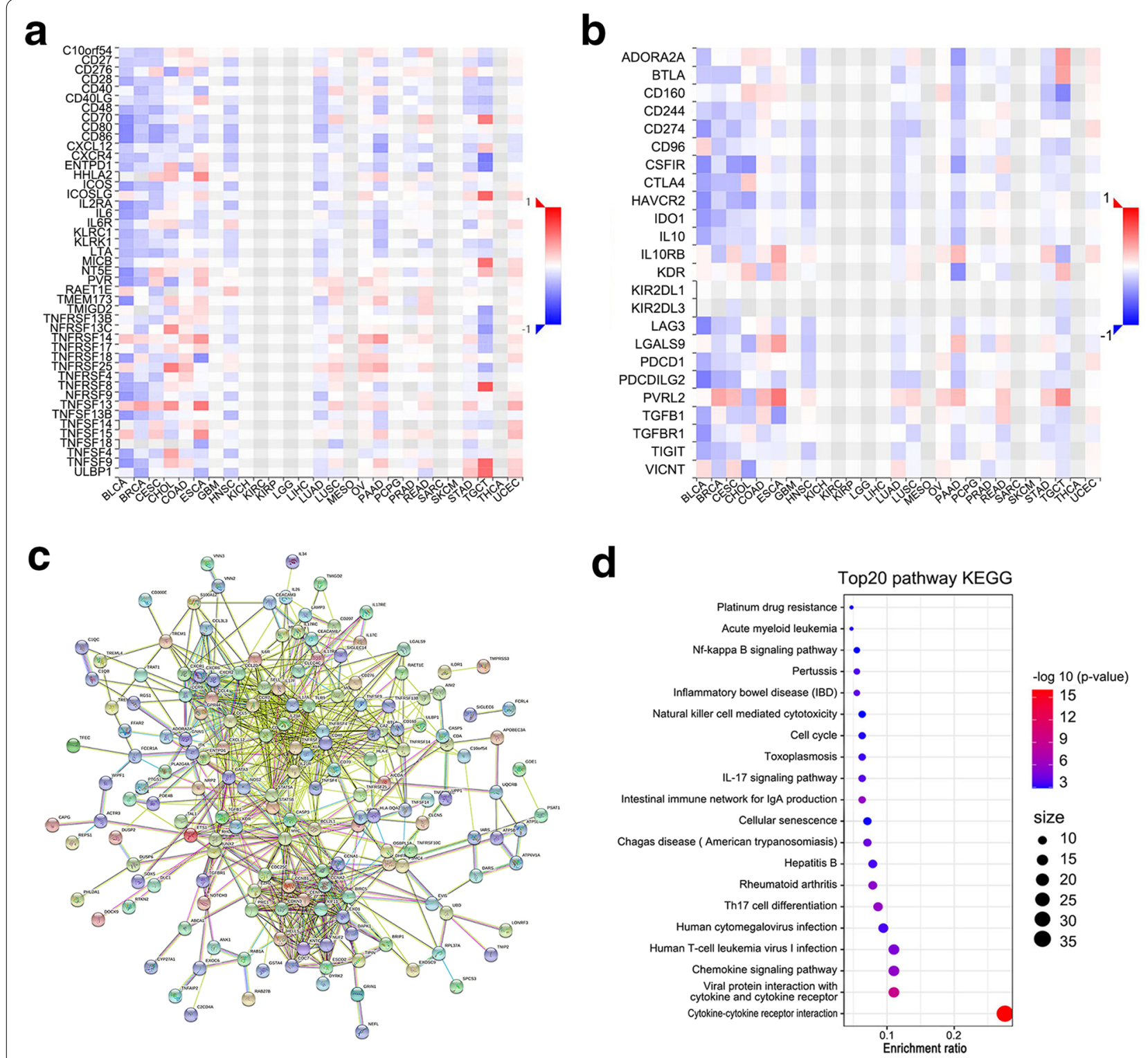

e
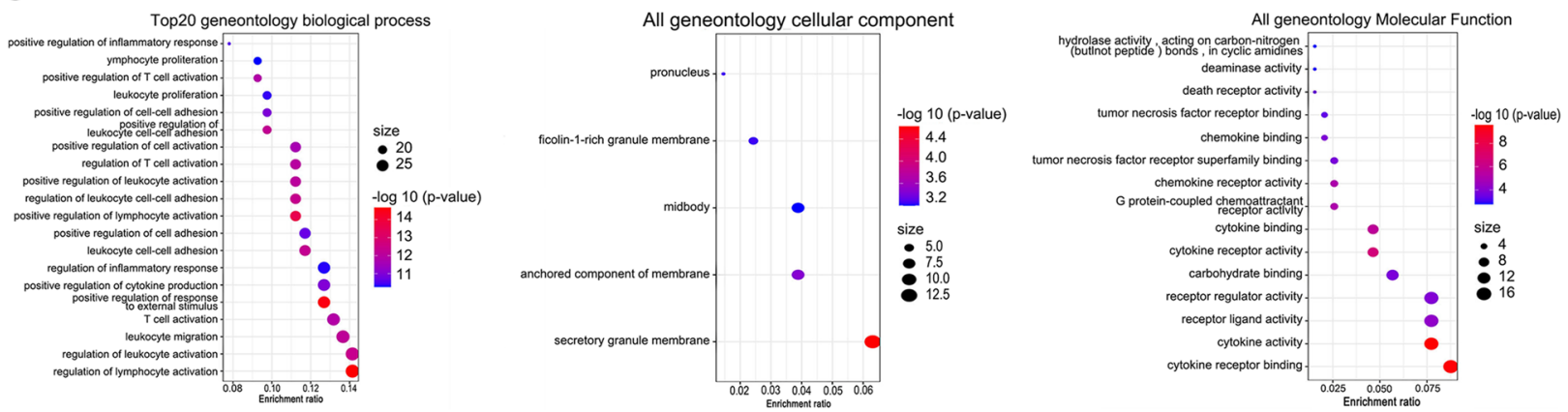

Fig. 6 Identification and analysis of immune signatures associated with SRMS. A The heatmap shows the correlation between SRMS and immune-stimulators. B The heatmap shows the correlation between SRMS and immune-inhibitors. C Protein-protein interaction network of SRMS-associated immunomodulators and related immune cell marker genes in CRC. $\mathbf{D}$ and $\mathbf{E}$ are the KEGG and GO of SRMS-associated immunomodulators and immune cell marker genes, respectively, where larger dot sizes are related with higher gene counts and a darker red color is correlated to lower $P$ value 


\section{Author details}

${ }^{1}$ Department of Clinical Laboratory, Xiangya Hospital, Central South University, 87 Xiangya Road, Kaifu District, Changsha 410008, China. ${ }^{2}$ Department of Essential Surgery, Xiangya Hospital, Central South University, Changsha, China.

Received: 13 April 2021 Accepted: 24 October 2021 Published online: 16 November 2021

\section{References}

1. Dekker E, Tanis P, Vleugels J, Kasi P, Wallace M. Colorectal cancer. Lancet (London, England). 2019;394(10207):1467-80.

2. Weng W, Feng J, Qin H, Ma Y. Molecular therapy of colorectal cancer: progress and future directions. Int J Cancer. 2015;136(3):493-502.

3. Pang S, Awi N, Armon S, Lim W, Low J, Peh K, et al. Current update of laboratory molecular diagnostics advancement in management of colorectal cancer (CRC). Diagnostics (Basel, Switzerland). 2019;10:1.

4. Jackstadt R, van Hooff S, Leach J, Cortes-Lavaud X, Lohuis J, Ridgway R, et al. Epithelial NOTCH signaling rewires the tumor microenvironment of colorectal cancer to drive poor-prognosis subtypes and metastasis. Cancer Cell. 2019;36(3):319-36 e317.

5. Kohmura N, Yagi T, Tomooka Y, Oyanagi M, Kominami R, Takeda N, et al. A novel nonreceptor tyrosine kinase, Srm: cloning and targeted disruption. Mol Cell Biol. 1994;14(10):6915-25.

6. Goel R, Miah S, Black K, Kalra N, Dai C, Lukong K. The unique N-terminal region of SRMS regulates enzymatic activity and phosphorylation of its novel substrate docking protein 1. FEBS J. 2013;280(18):4539-59.

7. Arbesú M, Maffei M, Cordeiro T, Teixeira J, Pérez Y, Bernadó P, Roche S, Pons M: The Unique domain forms a fuzzy intramolecular complex in Src family kinases. Structure (London, England : 1993) 2017, 25(4):630-640. e634

8. McClendon C, Miller W: Structure, function, and regulation of the SRMS tyrosine kinase. Int J Mol Sci 2020, 21(12).

9. Yoo M, Park J, Han H, Yun Y, Kang J, Choi D, Lee J, Jung J, Lee K, Kim K: Discovery of gastric cancer specific biomarkers by the application of serum proteomics. Proteomics 2017, 17(6).

10. Tang Z, Li C, Kang B, Gao G, Li C, Zhang Z. GEPIA: a web server for cancer and normal gene expression profiling and interactive analyses. Nucleic Acids Res. 2017:45:W98-W102.

11. Rhodes D, Kalyana-Sundaram S, Mahavisno V, Varambally R, Yu J, Briggs B, Barrette T, Anstet M, Kincead-Beal C, Kulkarni P et al: Oncomine 3.0: genes, pathways, and networks in a collection of 18,000 cancer gene expression profiles. Neoplasia (New York, NY) 2007, 9(2):166-180.

12. Pontén $\mathrm{F}$, Jirström $\mathrm{K}$, Uhlen $\mathrm{M}$. The human protein atlas--a tool for pathology. J Pathol. 2008;216(4):387-93.

13. Chandrashekar D, Bashel B, Balasubramanya S, Creighton C, PonceRodriguez I, Chakravarthi B, Varambally S: UALCAN: A Portal for Facilitating Tumor Subgroup Gene Expression and Survival Analyses. Neoplasia (New York, NY) 2017, 19(8):649-658.

14. Vasaikar S, Straub P, Wang J, Zhang B. LinkedOmics: analyzing multiomics data within and across 32 cancer types. Nucleic Acids Res. 2018;46:D956-63.

15. Ru B, Wong C, Tong Y, Zhong J, Zhong S, Wu W, Chu K, Wong C, Lau C, Chen l et al:TISIDB: an integrated repository portal for tumorimmune system interactions. Bioinformatics (Oxford, England) 2019 35(20):4200-4202

16. El-Arabey A, Abdalla M, Abd-Allah A. SnapShot: TP53 status and macrophages infiltration in TCGA-analyzed tumors. Int Immunopharmacol. 2020;86:106758.

17. Szklarczyk D, Gable A, Lyon D, Junge A, Wyder S, Huerta-Cepas J, et al. STRING V11: protein-protein association networks with increased coverage, supporting functional discovery in genome-wide experimental datasets. Nucleic Acids Res. 2019;47:D607-13.

18. Gocek E, Moulas A, Studzinski G. Non-receptor protein tyrosine kinases signaling pathways in normal and cancer cells. Crit Rev Clin Lab Sci. 2014;51(3):125-37.

19. Gilic M, Tyner A. Targeting protein tyrosine kinase 6 in cancer. Biochimica et biophysica acta Reviews on cancer. 1874;2020(2):188432.
20. Fan G, Aleem S, Yang M, Miller W, Tonks N. Protein-tyrosine phosphatase and kinase specificity in regulation of SRC and breast tumor kinase. J Biol Chem. 2015;290(26):15934-47.

21. Mathur P, Gierut J, Guzman G, Xie H, Xicola R, Llor X, et al. Kinase-dependent and -independent roles for PTK6 in colon cancer. Molecular cancer research : MCR. 2016;14(6):563-73.

22. Lauer S, Avecilla G, Spealman P, Sethia G, Brandt N, Levy S, et al. Singlecell copy number variant detection reveals the dynamics and diversity of adaptation. PLoS Biol. 2018;16(12):e3000069.

23. Tsai Y, Huang S, Lin H, Lin C, Lan Y, Wang H, et al. Differences in gene mutations according to gender among patients with colorectal cancer. World J Surg Oncol. 2018;16(1):128.

24. Marchetti M, Meloni M, Anwar M, Zen A, Sala-Newby G, Slater S, et al. MicroRNA-24-3p targets notch and other vascular morphogens to regulate post-ischemic microvascular responses in limb muscles. Int J Mol Sci. 2020;21:5.

25. Raafat A, Bargo S, McCurdy D, Callahan R. The ANK repeats of Notch-4/ Int3 activate NF-kB canonical pathway in the absence of Rbpj and causes mammary tumorigenesis. Sci Rep. 2017;7(1):13690.

26. Lu S, Dong W, Zhao P. Liu Z: IncRNA FAM83H-AS1 is associated with the prognosis of colorectal carcinoma and promotes cell proliferation by targeting the notch signaling pathway. Oncol Lett. 2018;15(2):1861-8.

27. Wozniak D, Kajdacsy-Balla A, Macias V, Ball-Kell S, Zenner M, Bie W, et al. PTEN is a protein phosphatase that targets active PTK6 and inhibits PTK6 oncogenic signaling in prostate cancer. Nat Commun. 2017;8(1):1508.

28. Gierut J, Zheng Y, Bie W, Carroll R, Ball-Kell S, Haegebarth A, et al. Disruption of the mouse protein tyrosine kinase 6 gene prevents STAT3 activation and confers resistance to azoxymethane. Gastroenterology. 2011;141(4):1371-80 1380.e1371-1372.

29. Tan M, Wong C, Tan H, Yang X, Ditlev J, Matsuda D, et al. Genomic expression and single-nucleotide polymorphism profiling discriminates chromophobe renal cell carcinoma and oncocytoma. BMC Cancer. 2010;10:196.

30. Chang $H$, Jeung $H$, Jung J, Kim T, Rha S, Chung H. Identification of genes associated with chemosensitivity to SAHA/taxane combination treatment in taxane-resistant breast cancer cells. Breast Cancer Res Treat. 2011;125(1):55-63.

31. Cai Y, Li Y, Shi C, Zhang Z, Xu J, Sun B. LncRNA OTUD6B-AS1 inhibits many cellular processes in colorectal cancer by sponging miR-21-5p and regulating PNRC2. Hum Exp Toxicol. 2021:960327121997976.

32. Williams K, Ghosh R, Giridhar P, Gu G, Case T, Belcher S, et al. Inhibition of stathmin 1 accelerates the metastatic process. Cancer Res. 2012;72(20):5407-17

33. Alli E, Bash-Babula J, Yang J, Hait W. Effect of stathmin on the sensitivity to antimicrotubule drugs in human breast cancer. Cancer Res. 2002;62(23):6864-9.

34. LvY, Zheng X, Shi M, Wang Z, Cui L. Different EPHX1 methylation levels in promoter area between carbamazepine-resistant epilepsy group and carbamazepine-sensitive epilepsy group in Chinese population. BMC Neurol. 2019;19(1):114.

35. Landry-Voyer A, Bergeron D, Yague-Sanz C, Baker B, Bachand F. PDCD2 functions as an evolutionarily conserved chaperone dedicated for the 405 ribosomal protein US5 (RPS2). Nucleic Acids Res. 2020;48(22):12900-16.

36. Orgebin E, Lamoureux F, Isidor B, Charrier C, Ory B, Lézot F, Baud'huin M: Ribosomopathies: New Therapeutic Perspectives. Cells 2020, 9(9).

37. Liu D, Lin L, Wang Y, Chen L, He Y, Luo Y, et al. PNO1, which is negatively regulated by miR-340-5p, promotes lung adenocarcinoma progression through notch signaling pathway. Oncogenesis. 2020;9(5):58.

38. Zhao Y, Yang $X, X u X$, Zhang J, Zhang L, Xu H, et al. Deubiquitinase PSMD7 regulates cell fate and is associated with disease progression in breast cancer. Am J Transl Res. 2020;12(9):5433-48.

39. Moreno-Sánchez R, Rodríguez-Enríquez S, Saavedra E, Marín-Hernández A, Gallardo-Pérez J. The bioenergetics of cancer: is glycolysis the main ATP supplier in all tumor cells? BioFactors (Oxford, England). 2009:35(2):209-25.

40. Huang Q, Chen Z, Cheng P, Jiang Z, Wang Z, Huang Y, et al. LYRM2 directly regulates complex I activity to support tumor growth in colorectal cancer by oxidative phosphorylation. Cancer Lett. 2019;455:36-47. 
41. Wang Y, Wang J, Yang C, Wang Y, Liu J, Shi Z, et al. A study of the correlation between $\mathrm{M} 2$ macrophages and lymph node metastasis of colorectal carcinoma. World J Surg Oncol. 2021;19(1):91.

42. Tosolini M, Kirilovsky A, Mlecnik B, Fredriksen T, Mauger S, Bindea G, et al. Clinical impact of different classes of infiltrating $T$ cytotoxic and helper cells (Th1, th2, treg, th17) in patients with colorectal cancer. Cancer Res. 2011;71(4):1263-71.

43. Dumitru C, Lang S, Brandau S. Modulation of neutrophil granulocytes in the tumor microenvironment: mechanisms and consequences for tumor progression. Semin Cancer Biol. 2013;23(3):141-8.

44. Zitvogel L, Tesniere A, Kroemer G. Cancer despite immunosurveillance: immunoselection and immunosubversion. Nat Rev Immunol. 2006;6(10):715-27.

45. Shi Y, Zhao Q, Liu X, Dong S. E J, Li X, Liu C, Wang H: toll-like receptor 4 regulates spontaneous intestinal tumorigenesis by up-regulating $\mathrm{LL}-6$ and GM-CSF. J Cell Mol Med. 2020;24(1):385-97.

46. Zhao J, Ou B, Han D, Wang P, Zong Y, Zhu C, et al. Tumor-derived CXCL5 promotes human colorectal cancer metastasis through activation of the ERK/EIK-1/snail and AKT/GSK3// $\beta$-catenin pathways. Mol Cancer. 2017;16(1):70
47. Do H, Lee C, Cho J. Chemokines and their receptors: multifaceted roles in cancer progression and potential value as cancer prognostic markers. Cancers (Basel). 2020;12:2

48. Yang Z, Tao Y, Xu X, Cai F, Yu Y, Ma L. Bufalin inhibits cell proliferation and migration of hepatocellular carcinoma cells via APOBEC3F induced intestinal immune network for IgA production signaling pathway. Biochem Biophys Res Commun. 2018;503(3):2124-31.

49. Housseau F, Wu S, Wick E, Fan H, Wu X, Llosa N, et al. Redundant innate and adaptive sources of IL17 production drive colon tumorigenesis. Cancer Res. 2016;76(8):2115-24.

50. Werner J, Gessner M, Lilly L, Nelson M, Metz A, Horn D, et al. Neutrophils produce interleukin 17A (IL-17A) in a dectin-1- and IL-23-dependent manner during invasive fungal infection. Infect Immun. 2011;79(10):3966-77.

\section{Publisher's Note}

Springer Nature remains neutral with regard to jurisdictional claims in published maps and institutional affiliations.
Ready to submit your research? Choose BMC and benefit from:

- fast, convenient online submission

- thorough peer review by experienced researchers in your field

- rapid publication on acceptance

- support for research data, including large and complex data types

- gold Open Access which fosters wider collaboration and increased citations

- maximum visibility for your research: over $100 \mathrm{M}$ website views per year

At BMC, research is always in progress.

Learn more biomedcentral.com/submissions 